\title{
Homeobox genes d11-d13 and a13 control mouse autopod cortical bone and joint formation
}

\author{
Pablo Villavicencio-Lorini,1,2 Pia Kuss,,1,2 Julia Friedrich,,1,2 Julia Haupt,1,2 Muhammed Farooq, ${ }^{3}$ \\ Seval Türkmen, ${ }^{2}$ Denis Duboule, ${ }^{4}$ Jochen Hecht, ${ }^{1,5}$ and Stefan Mundlos ${ }^{1,2,5}$ \\ ${ }^{1}$ Max Planck Institute for Molecular Genetics, Berlin, Germany. ${ }^{2}$ Institute for Medical Genetics, Charité, Universitätsmedizin Berlin, Berlin, Germany. \\ ${ }_{3}^{3}$ Human Molecular Genetics Laboratory, National Institute for Biotechnology \& Genetic Engineering (NIBGE), Faisalabad, Pakistan. \\ ${ }^{4}$ National Research Centre Frontiers in Genetics, Department of Zoology and Animal Biology, University of Geneva, Geneva, Switzerland. \\ ${ }^{5}$ Berlin-Brandenburg Center for Regenerative Therapies (BCRT), Charité, Universitätsmedizin Berlin, Berlin, Germany.
}

\begin{abstract}
The molecular mechanisms that govern bone and joint formation are complex, involving an integrated network of signaling pathways and gene regulators. We investigated the role of Hox genes, which are known to specify individual segments of the skeleton, in the formation of autopod limb bones (i.e., the hands and feet) using the mouse mutant synpolydactyly homolog $(s p d h)$, which encodes a polyalanine expansion in Hoxd13. We found that no cortical bone was formed in the autopod in $s p d h / s p d h$ mice; instead, these bones underwent trabecular ossification after birth. $S p d h / s p d h$ metacarpals acquired an ovoid shape and developed ectopic joints, indicating a loss of long bone characteristics and thus a transformation of metacarpals into carpal bones. The perichondrium of $s p d h / s p d h$ mice showed abnormal morphology and decreased expression of Runt-related transcription factor 2 (Runx2), which was identified as a direct Hoxd13 transcriptional target. Hoxd11 ${ }^{-/}$Hoxd12 $2^{-/}$Hoxd13 $3^{-/}$triple-knockout mice and Hoxd $13^{-/-}$Hoxa $13^{+/-}$mice exhibited similar but less severe defects, suggesting that these Hox genes have similar and complementary functions and that the $s p d b$ allele acts as a dominant negative. This effect was shown to be due to sequestration of other polyalanine-containing transcription factors by the mutant Hoxd13 in the cytoplasm, leading to their degradation. These data indicate that Hox genes not only regulate patterning but also directly influence bone formation and the ossification pattern of bones, in part via Runx2.
\end{abstract}

\section{Introduction}

The molecular mechanisms that govern bone formation are complex, but, based on genetic studies mainly in humans and mice, a network of important players that control individual steps of this process has emerged $(1,2)$. The early pattern step is determined by a number of signaling pathways, including the families of Homeobox (Hox), Sonic hedgehog (Shb), Fgf, and Wingless related (Wnt) genes. Thereafter, mesenchymal progenitor cells begin to adhere to each other and condense to form a template anlage of the future bone. In those bones that undergo endochondral ossification, chondrocytes in the middle of the anlage hypertrophy and mineralize; blood vessels invade, and the cartilage is removed and replaced by trabecular bone. At the same time, a bone shaft that will become the future cortical bone is formed surrounding the cartilaginous template. This part of the bone is derived from perichondrial cells that differentiate directly into osteoblasts, a process that is initiated and controlled by a series of transcription factors, of which Runt-related transcription factor $2(R u n x 2)$ is probably the most important, as shown by gene inactivation experiments in mice (3).

The initial process of bone formation that takes place in the middle of the bone anlage is generally referred to as the primary ossification center. Secondary ossification centers form in all long bones at later time points (usually after birth) in the cartilaginous ends of the bones. In these areas, separated from the primary ossification

Authorship note: Pablo Villavicencio-Lorini and Pia Kuss contributed equally to this work.

Conflict of interest: The authors have declared that no conflict of interest exists. Citation for this article: J Clin Invest. 2010;120(6):1994-2004. doi:10.1172/JCI41554. center through the growth plate, chondrocytes become hypertrophic, and their matrix mineralizes, is removed, and finally replaced by trabecular bone. The process is similar to that taking place in the primary ossification center, with the difference that no cortical bone is formed. Besides in the cartilaginous ends of long bones, trabecular ossification takes place in the carpal bones, which form the wrist in humans. The molecular processes that control primary versus secondary (trabecular) ossification are poorly understood.

Homeobox genes and the proteins they encode, the homeodomain proteins, play important roles in the developmental processes of many multicellular organisms. A subgroup of these genes, the Hox genes, are found in clusters on the chromosomes. Within a cluster, the genes are arranged in the order of their function along the anteroposterior body axis (4). In mammals, 4 such clusters exist, which harbor a total of 39 genes. During development of the limbs, Hox genes of the A and D clusters, and in particular those of the $5^{\prime}$ end, i.e., Hoxa10, -a11, -a13, Hoxd9, -d10, -d11, -d12, $-d 13$, are of particular importance. The critical importance of Hox genes for normal growth and patterning of the autopod skeleton has been established via loss and gain of function manipulations in the mouse (5). Combinations of Hox proteins are thought to specify individual segments of the appendicular skeleton. In the hind limb, Hox10 paralogous genes are required to pattern the stylopod, and Hox11 paralogous genes are required to pattern the zeugopod (6). Hox13 paralogous genes were shown to be important for patterning of the autopod (7). Overall, loss-of-function mutations in Hox genes appear to systematically involve loss or reduction of skeletal elements, and Hox gene function has accordingly been interpreted to specify individual segments of the limb in a proximo-distal fashion. 
Mutations in HOX genes result in human phenotypes. Expansions of an alanine (Ala) repeat in HOXD13, for example, cause synpolydactyly, a limb malformation characterized by an additional digit between digits 3 and 4 and a fusion (syndactyly) among these digits (8). The nature of this mutation has been studied in vitro and in a mouse mutant that carries the same mutation and hence was named synpolydactyly homolog $(s p d b)(9,10)$. The polyalanine expansions in the human and mouse mutants result in intracellular aggregation and subsequent degradation of the mutant protein (11). This is likely to result in a dominant negative effect, as mice with inactivated Hoxd13 alleles do not show synpolydactyly $(12,13)$, but homozygous deficiency for Hoxd11, Hoxd12, and Hoxd13 is associated with central polydactyly similar to human synpolydactyly (14).

In this study, we focused on the process of bone formation in $s p d h$ mutant and Hox knockout mice. We show that $s p d h / s p d h$ mice do not form cortical bone in the autopod. The perichondrium is insufficiently differentiated, and the absence of bone formation is accompanied by a loss of Runx 2 expression. Runx2, in turn, is directly regulated by Hoxd13, indicating that Hox genes in the limb are upstream of Runx2. Although cortical bone is not formed, bone formation takes place after birth by secondary ossification of hypertrophic cartilage. Furthermore, the mutant metacarpals become more round in shape and form joint cartilage at their sides, thus mimicking the morphology of carpal bones. Our results show that Hox genes are important for bone formation in the limbs and determine the ossification pattern of bones.

\section{Results}

Abnormal ossification in spdh and Hox mutant mice. The synpolydactyly homolog mouse $s p d h$ carrying a homozygous expansion mutation with 7 additional alanines in the $\mathrm{N}$-terminal polyalanine repeat of Hoxd13 showed a severely disturbed differentiation process of the distal skeletal elements. In newborn $s p d h / s p d h$ mice, polydactyly and missing ossification centers were apparent (Figure 1A). In contrast to control mice, there was no proper separation of digital elements into metacarpals and phalanges, as no joints were formed. In areas in which Hoxd13 is not expressed, i.e., in the stylopod and zeugopod, the skeleton was normal. At P4, Alizarin Red positive zones appeared, with an irregular shape and distribution corresponding to mineralized hypertrophic chondrocytes. These areas became broader at P7 and expanded over the entire distal forelimb. By P14, the entire autopod skeleton was mineralized. We investigated the metacarpal bones in more detail to analyze the process of mineralization and ossification (Figure 1B). Control mice at P0 showed cortical bone in the middle and trabecular bone at the ends, near the growth plates. By P7, this zone had expanded and additional zones of mineralization that would become the later secondary ossification centers appeared at the distal end. In $s p d h / s p d h$ mice, no mineralization was present at $\mathrm{P} 0$. At P7, mineralized cartilage was observed, but no cortical bone. At P14, most of the mineralized cartilage had been removed and replaced by trabecular bone.

In previous reports, it was shown that a triple inactivation of Hoxd11, Hoxd12, and Hoxd13 results in a synpolydactyly phenotype (14). We investigated mineralization and ossification in these mice. As shown in Figure 1, A and B, Hoxd11,12,13 del/del mice exhibit defect similar to that of $s p d h / s p d h$ mice. There was no mineralization at P0. At P7, most metacarpals were mineralized, but the majority of mineral was present within the chondrocytes. The overall phenotype, however, was milder than in $s p d h / s p d h$ mice. We observed that centers of mineralization appeared earlier (at P2) in the Hoxd11,12,13 del/del mice than in $s p d h / s p d h$ mice.

To compare the effect of the mutation with the effect of lack of single Hox genes, we investigated mice with other inactivated Hox genes (Figure 1C). Inactivation of Hoxd13 results in a relatively mild phenotype consisting of an overall reduction in size and abnormal metacarpophalangeal joints, but pentadactyly (13). In digit 2, however, abnormal ossification was present in the proximal part of the metacarpal. Here, a pattern similar to that observed in the $s p d h / s p d h$ mice was observed, resulting in a bone that, at least partially, consisted of trabecular bone only. Hoxa13 is largely coexpressed with Hoxd13 in the distal limb. We therefore investigated possible genetic interactions among the spdh allele, Hoxd13, and Hoxa13. Mice with inactivated Hoxd13 and 1 inactivated Hoxa13 allele (Hoxd13--; Hoxa13 $\left.{ }^{+-}\right)$showed a severe phenotype with very small autopods consisting of 6 digits without joints as previously reported $(7,15)$. Similar to the $s p d h / s p d h$ mutants, these mice showed a complete lack of mineralization at P0. At P7, mineralization was present, but only within the cartilage anlagen. No cortical bone was identified. Spdh/spdh mice with 1 inactivated Hoxa13 allele (spdh/spdh; Hoxa13 $\left.{ }^{+/-}\right)$showed the most severe phenotype. We observed a fusion of all cartilaginous elements that were greatly reduced in size. Mineralization was highly abnormal, showing only mineralized cartilage and no cortical bone formation.

Abnormal perichondrium in spdh mutant mice. Based on our previous finding that $s p d h / s p d h$ mice show narrow interdigital spaces with partially fused cartilaginous rudimentary fingers and no clear border formation, we characterized the perichondrial layer in more detail. WT mice showed a broad peridigital expression of Hoxd13 protein with enhancement in the perichondrial layer. In $s p d h / s p d h$ mice, Hoxd13 protein was reduced and showed an abnormal distribution (Figure 2A). Histological analysis of the perichondrium in WT mice showed the typical elongated fibroblast-like cells around the cartilage anlagen that form the perichondrium and, at a later stage, the periosteum. These cells were apparently absent in $s p d h / s p d h$ mice. Periostin, a gene predominantly expressed in the periosteum and perichondrium, and collagen type 2 alpha 1 (Col2a1) showed a mutually exclusive expression pattern in WT mice. In spdh mice, the expression patterns were overlapping, resulting in fuzzy contours (Supplemental Figure 1; supplemental material available online with this article; doi:10.1172/JCI41554DS1). Furthermore, chondrocytes as well as perichondrial cells had lost their polarity (Figure 2B).

Perichondrial cells express a number of genes that correspond to the different layers of cell differentiation within this tissue. Collagen type 1 alpha 1 (Col1a1) was strongly upregulated in perichondrial cells in WT (Figure 2, C and D). In spdh/spdh mice, we observed low Col1a1 expression in the interdigital space and very little expression in the cells adjacent to the chondrocytes. The same was shown for collagen type 1 protein. Other genes known to be expressed in the perichondrium were also downregulated. These included periostin (Postn) (16), Wnt5a (17), cellular retinoic acid binding protein 1 ( Crabp1), and tumor suppressor region 2 protein (Tsp2) (18), as shown by in situ hybridization (Figure 2E).

To determine whether these genes are regulated by Hoxd13, we infected chicken micromass cells (chMM) plated at high density with a RCASBP retrovirus (replication-competent ASLV [avian sarcoma-leukosis virus] long terminal repeat [LTR] with a splice acceptor and Bryan polymerase) expressing WT and mutant Hoxd13. mRNA levels were quantified and compared with control levels (empty virus). As shown in Figure 2F, Hoxd13 WT expression upregulated the perichondrially expressed genes Tsp2, Postn, 
A
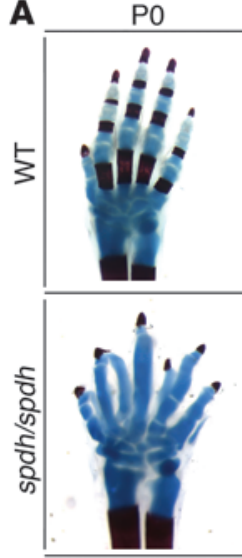

P4

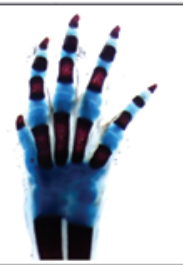

P7
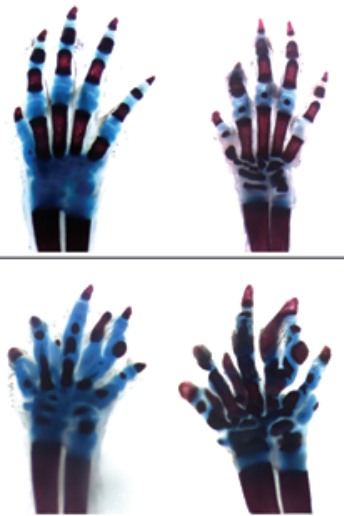

P14
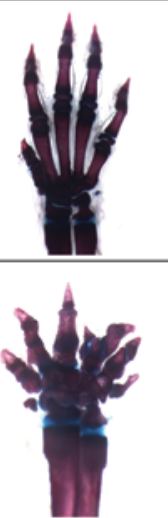
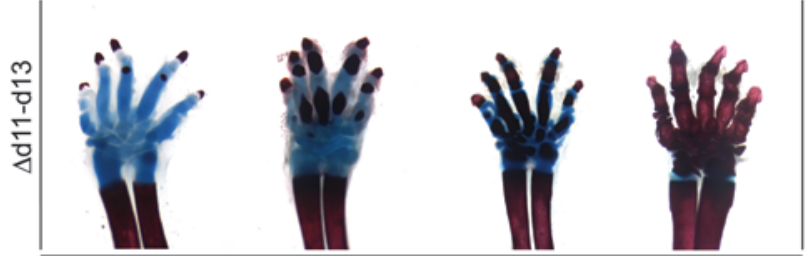

C
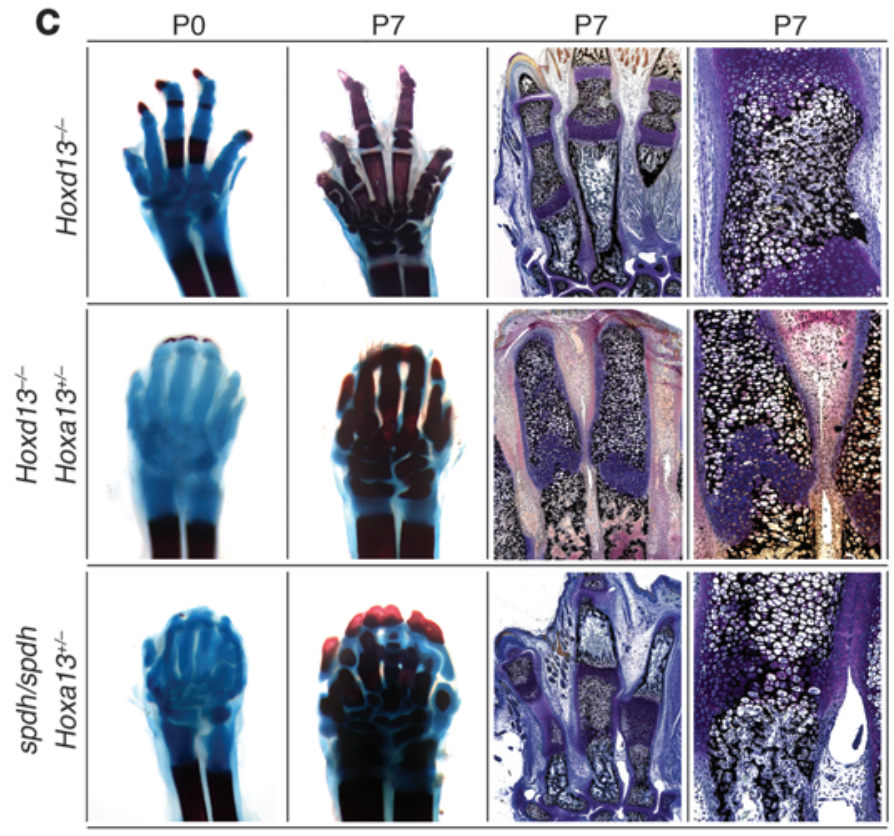

B

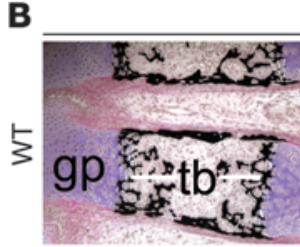

$\mathrm{PO}$
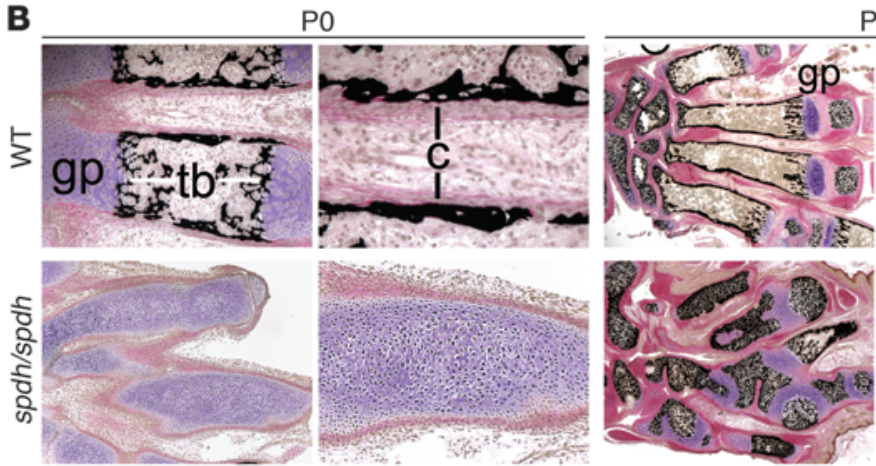

P7
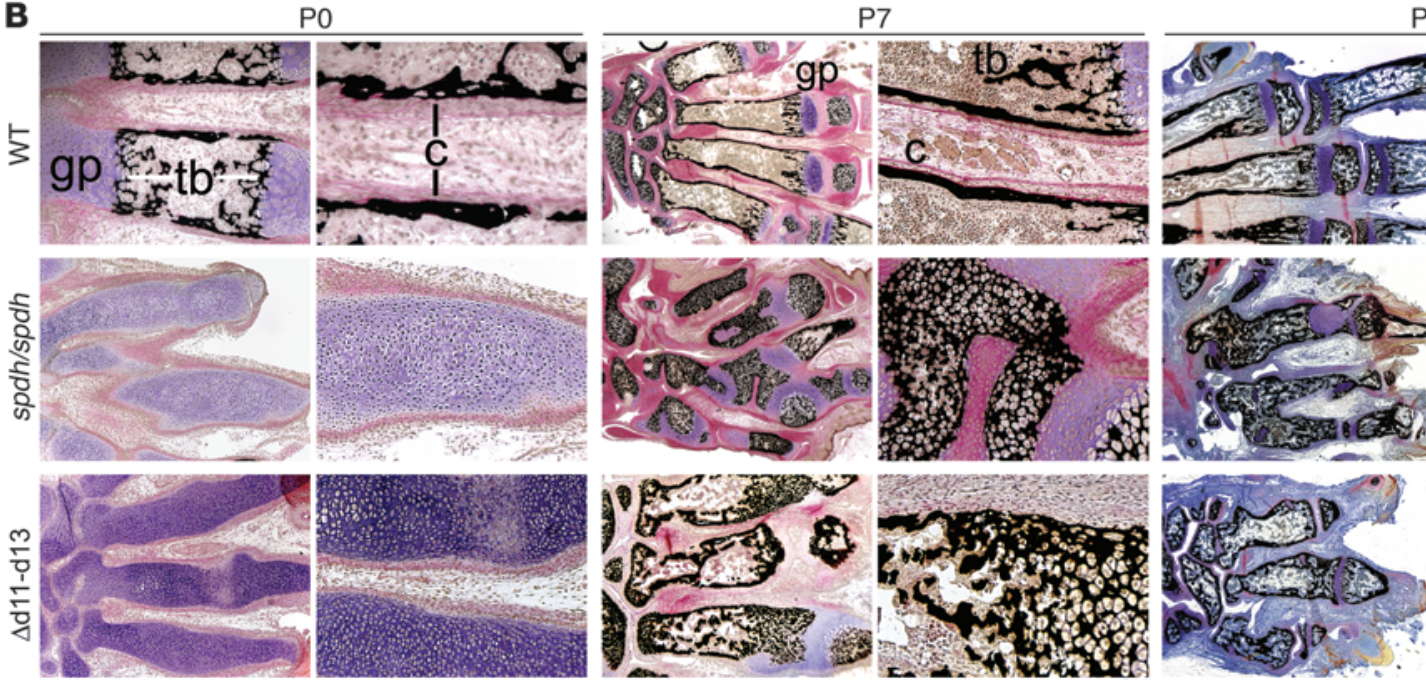

P14
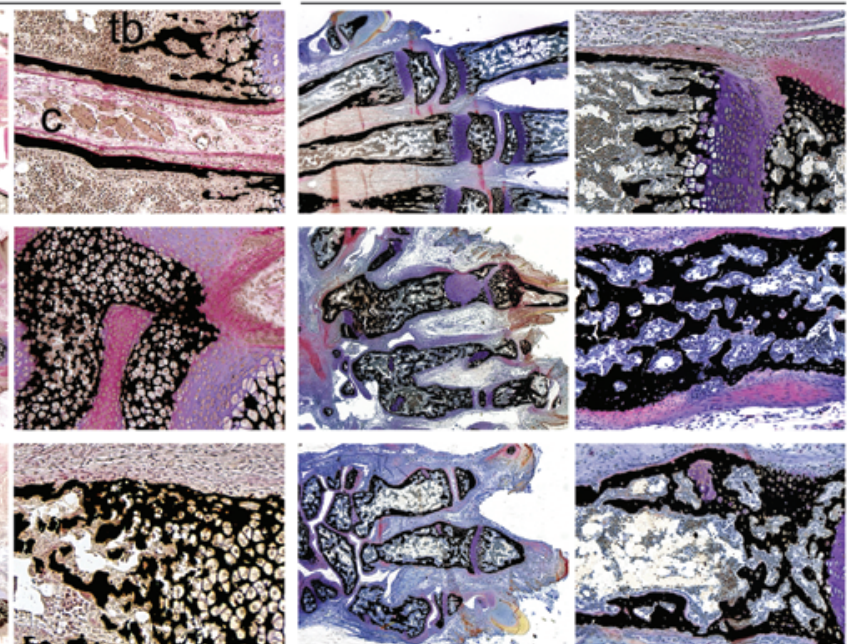

Figure 1

Skeletal phenotype of Hox mutant mice. (A) Skeletal preparations of postnatal stages P0, P4, P7, and P14 of WT, spdh/spdh, and Hoxd11,12,13delddel ( $\Delta$ d11-d13) mice. Cartilage stained with Alcian Blue, bone with Alizarin Red. Note additional digit-like structures, a severe delay in ossification, and missing joints in mutant mice. (B) Histology (von Kossa) of postnatal stages P0, P7, and P14 of WT, spdh/spdh, and Hoxd11,12,13del/del ( $\Delta$ d11-d13) metacarpals. Mineralized regions are black, counterstaining with Toluidine Blue and Picro Fuchsin Red. At PO, WT mice show both cortical (C) and trabecular bone (tb) and adjacent growth plates (gp). In mutant mice, mineralization and hypertrophic chondrocytes are completely missing. At P7 in WT, secondary ossification centers are visible at the distal ends of metacarpals. In mutant mice, hypertrophic chondrocytes and mineralized cartilage are present but no cortical bone. At P14 in WT, mineralized cartilage of secondary ossification centers has been replaced by trabecular bone; cortical bone has expanded. In spdh/spdh and Hoxd11,12,13del/del ( $(\mathrm{d} 111-\mathrm{d} 13)$ mutants, growth plates are disorganized; mineralized regions have partly been replaced by trabecular bone. No cortical bone is present. Original magnification, $\times 200$. (C) Skeletal preparations at P0 and P7, histology

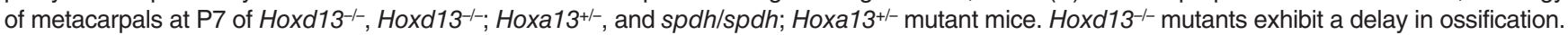
Hoxd13--; Hoxa13 ${ }^{+/-}$mice show a severe ossification defect at P0 and abnormal bone formation at P7 similar to the spdh/spdh mice. Spdh/spdh; Hoxa13+/- mutant mice show a similar but more severe phenotype.

and Wnt5a and, in a moderate manner, the genes Col1a1 and Crabp1. Mutant Hoxd13 with an alanine expansion (+7 Ala and +14 Ala), in contrast, resulted in no significant increase or even in a suppression. Protein expression of WT and mutant Hoxd13 was verified on Western blot with anti-FLAG or anti-Hoxd13 anti- bodies. Other markers that are expressed by preosteoblasts, such as osteopontin, were not detectable in $s p d h / s p d h$ mice, indicating the absence of osteoblasts and their precursors (not shown).

Hox genes regulate Runx2. Runx2 is a transcription factor that is essential for bone formation (3). We therefore investigated 
A

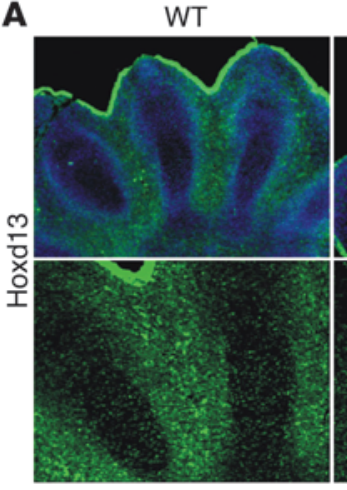

C

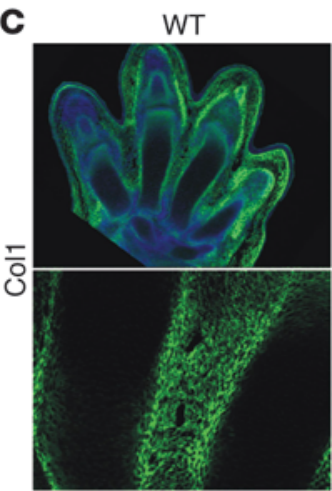

E

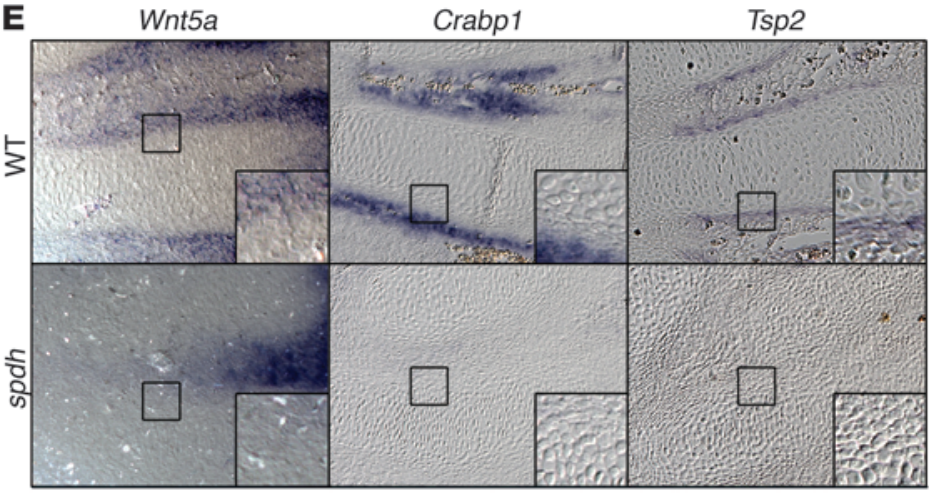

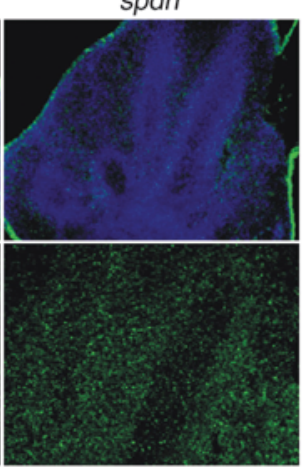

spdh

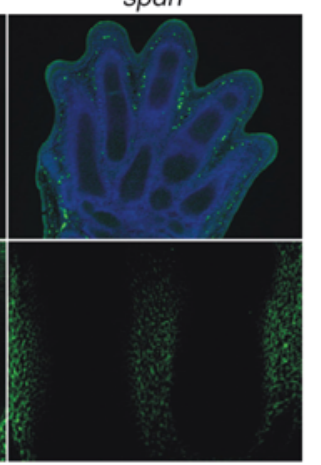

Crabp1
B

$F$

WT
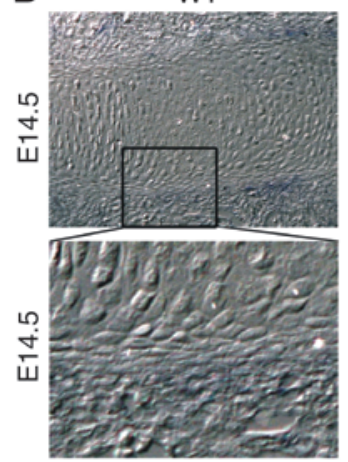

D
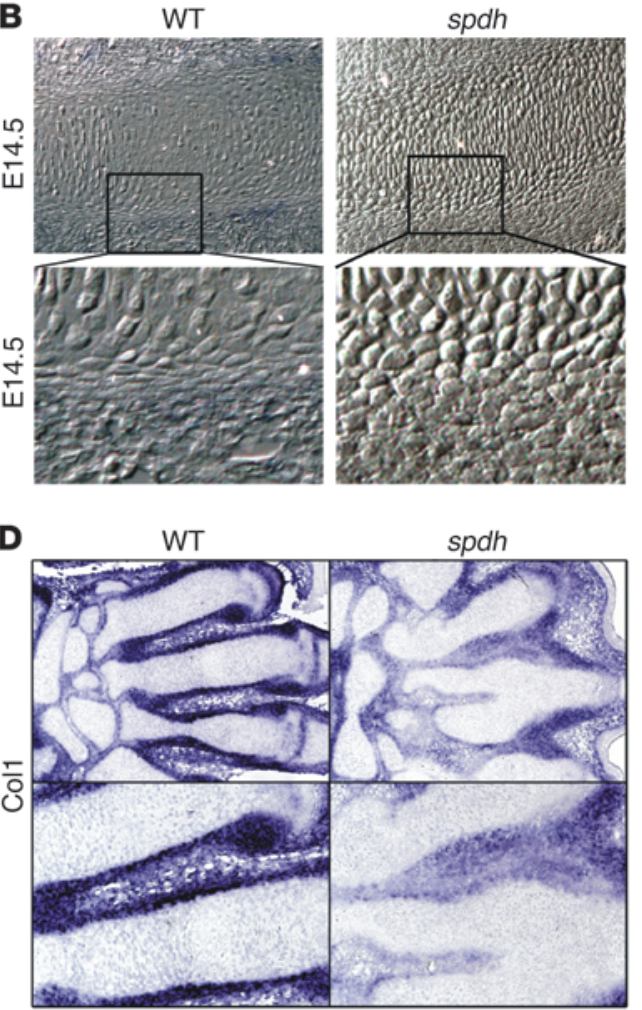

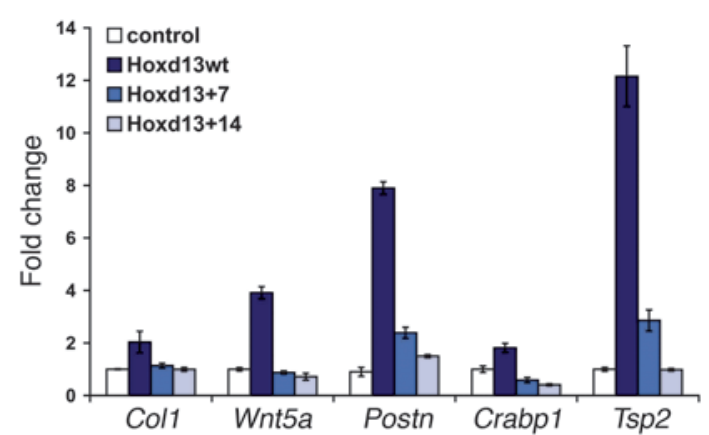

Figure 2

Abnormal perichondrium in spdh/spdh mice. (A) Immunohistochemistry of WT and spdh forelimbs at E13.5. Hoxd13 protein (green) shows abnormal distribution and reduced amount in spdh/spdh mice. Lower panel shows magnification without DAPI merge. Original magnification, $\times 200$ (lower panels). (B) Morphology of perichondrial regions at E14.5 in WT and spdh/spdh mice (Nomarski interference contrast). Chondrocytes of spdh/spdh mice show no apparent orientation, and the flattened perichondrial cells are missing. (C) Immunolocalization and (D) in situ hybridization of Col1a1 on sections of WT and spdh/spdh metacarpals at E13.5. Col1 protein as well as mRNA is greatly reduced in mutants. Magnifications shown in the lower panel. (E) In situ hybridizations of perichondrially expressed genes Wnt5a, Crabp1, and Tsp2 at E14.5 in WT and spdh/spdh metacarpals. Note strong downregulation in spdh/spdh animals. Panels show a higher magnification of the perichondrium. Original magnification, $\times 400$ (panels); $\times 1000$ (insets). (F) Expression of WT but not mutant Hoxd13 in chMM results in the upregulation of perichondrially expressed genes Col1a1, Wnt5a, Postn, Crabp1, and Tsp2. Fold change values are referred to control for each gene. Data represent mean \pm SD.

the expression of Runx2 in the metacarpals of $s p d h / s p d h$ mice. As shown in Figure 3A, Runx2 was expressed in the perichondrium/ periosteum of E13.5 metacarpals and phalanges but not the carpals of WT autopods. In contrast, $s p d h / s p d h$ mice showed strongly reduced levels of Runx2 expression. This reduction was most pronounced in the metacarpal region and corresponded well with the complete lack of cortical bone in this region. To determine whether the trabecular bone formation observed at later post- natal stages escapes this apparent lack of Runx2 expression, we performed in situ hybridization of Runx2 on P7 limbs (Supplemental Figure 2). We observed Runx2 expression in hypertrophic chondrocytes and osteoblasts, indicating that Runx2 is initiated with the onset of hypertrophy but is extremely delayed.

Two color in situ hybridizations at stages E13.5 (Figure 3B) demonstrated coexpression of Hoxd13 and Runx2 in perichondrial cells. We therefore tested to determine whether Hoxd13 and other 
A

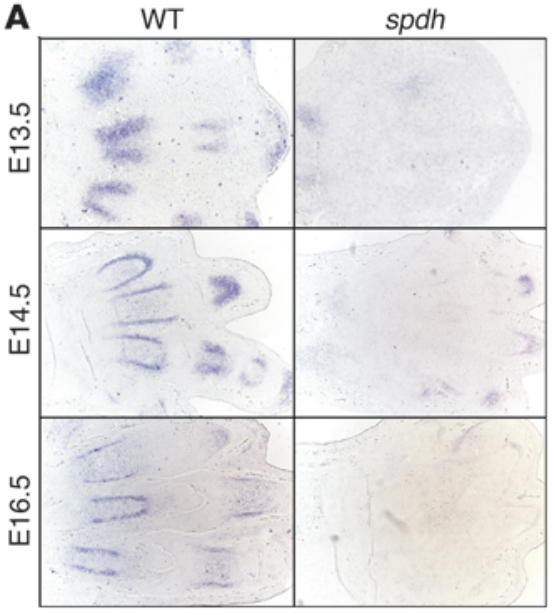

B

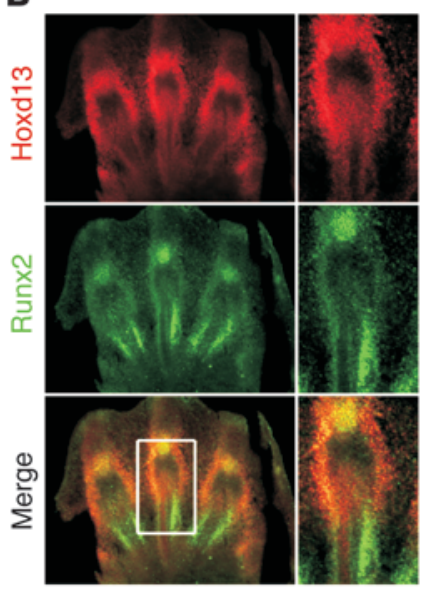

C

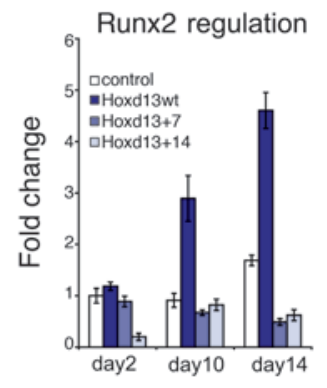

$\mathbf{F}$

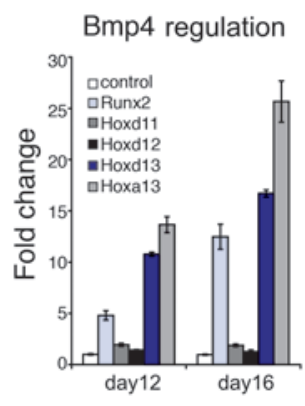

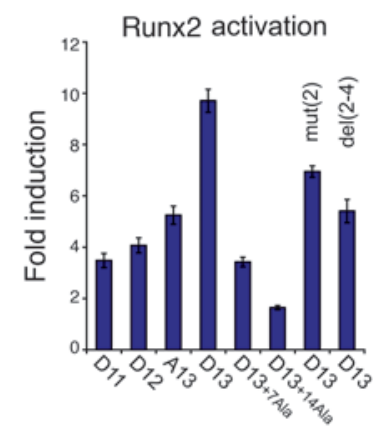

G

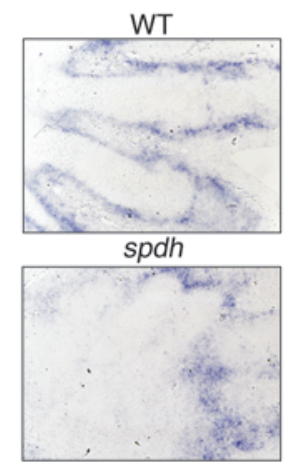

D

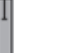

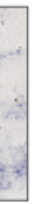

E

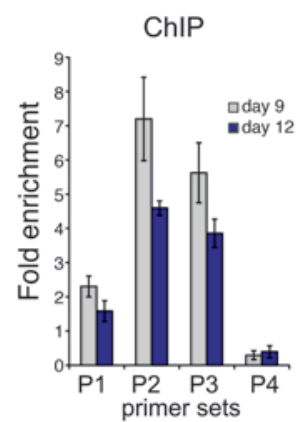

Runx2 Luciferase promoter Exon1

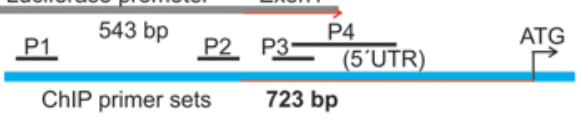

\section{Figure 3}

Hox genes regulate Runx2. (A) In situ hybridization with specific probe against Runx2 on WT and spdh/spdh limbs at E13.5, E14.5, and E16.5. Note strongly reduced expression in mutant mice. (B) Double fluorescent in situ hybridization for Hoxd13 (red) and Runx2 (green) on E13.5 WT limbs. Hoxd13 expression overlaps with Runx2 in the perichondrium. (C) qPCR analysis of chMM. Expression of WT Hoxd13, but not of mutant Hoxd13 (+7 and +14Ala) in chMM results in upregulation of Runx2. Fold changes refer to control at day 2. (D) Luciferase promoter assay shows activation of P1 promoter of Runx2 by WT but not by mutant Hoxd13. Activation is achieved in a lesser degree by Hoxd11, Hoxd12, and Hoxa13. Mutation of a putative Hoxd13-binding site at position -90 (mut[2]) and deletion of putative binding sites at -90, -78, and -63 (del[2-4]) decreases the Hoxd13 effect. Fold induction reflects the ratio of activation by gene of interest versus activation by empty vector in respective promoter constructs. (E) ChIP of Runx2 promoter P1 in chMM expressing Hoxd13. Enrichment with primer pairs P1, P2, and P3 but not P4. Position of primers is given in the schematic to the right. (F) Hoxd13 and Hoxa13 regulate Bmp4 in chMM. Fold changes refer to control at day 12. (G) Expression of Bmp4 in WT and spdh/spdh metacarpals at E14.5. Data in C, D, E, and F represent mean \pm SD.

autopod-expressed Hox genes are capable of inducing Runx2 in mesenchymal progenitor cells. We infected chMM with a RCASBP retrovirus containing WT or mutant Hoxd13 and quantified mRNA levels by quantitative PCR (qPCR). As shown in Figure 3C, Hoxd13 expression by RCASBP in chMM resulted in an upregulation of Run $x 2$ mRNA. The expression of the +14 Ala Hoxd13 mutant resulted in a suppression of Runx 2 compared with control infected cells.

To determine whether Runx2 is directly regulated by Hox genes, we performed promoter luciferase assays using a $0.543-\mathrm{kb}$ fragment of Runx2 exon 1 promoter (19) (Figure 3D). We found that Hoxd13 strongly activated the promoter, whereas the +7 Ala mutant resulted in less activation and the +14 Ala mutant showed no significant induction. In addition, Hoxa13, and to a lesser degree Hoxd11 and Hoxd12, showed induction of the Runx2 promoter. To determine whether Hoxd13 is able to directly bind to the Runx2 exon 1 promoter, we performed ChIP on chMM cells infected with a Hoxd13expressing retrovirus (Figure 3E). After immunoprecipitation, binding of Hoxd13 to the Runx2 promoter was determined by qPCR using primer pairs P1 to P4. We detected a significant enrichment for regions of the Runx2 promoter that are flanked by primer pairs P2 and P3, with close position to the assumed transcriptional start site, and to a lesser extent for the amplicon of P1, but not for the control (P4). The Hoxd13-binding matrix from Berger et al. (20) was used to identify potential Hoxd13-binding sites within the fragment of the mouse distal Runx 2 promoter. Five potential binding sites were found, at positions $-380,-90,-78,-63$, and +47 relative to the transcription start site. Deletion of binding sites 2-4 decreased induction of the Runx 2 promoter by Hoxd 13 to approximately $60 \%$ of the WT promoter (Figure 3D). Mutations of single binding sites $1,3,4$, or 5 had no significant effect on Hoxd13-mediated activation of the Runx2 promoter, whereas mutation of binding site 2 at position -90 reduced promoter activity by approximately $25 \%$. Binding sites 2-4 lie within a conserved region and can be found in the chicken Runx2 promoter as well. This region is enriched in ChIP using chMM. EMSAs showed binding of Hoxd13 to oligos containing binding sites 2-4 and 5 (data not shown). 
A

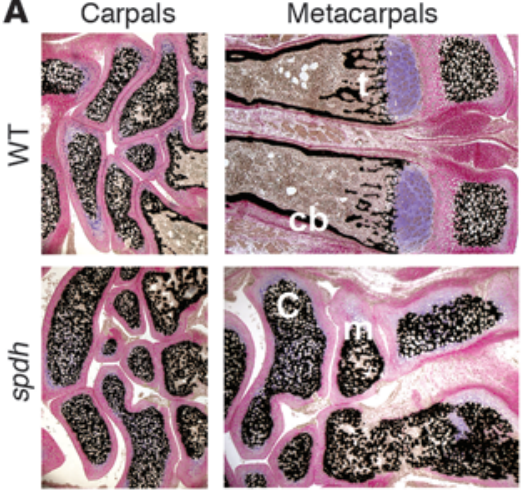

B
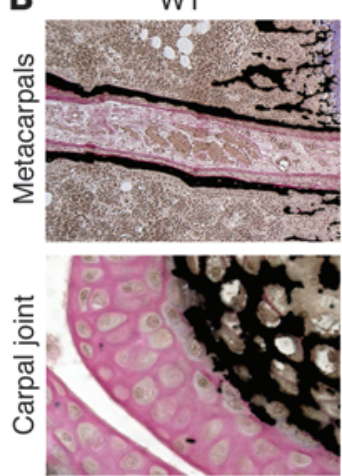

D

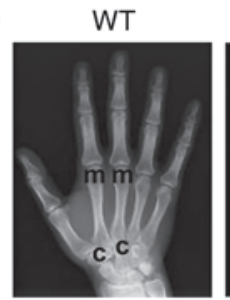

P7

C

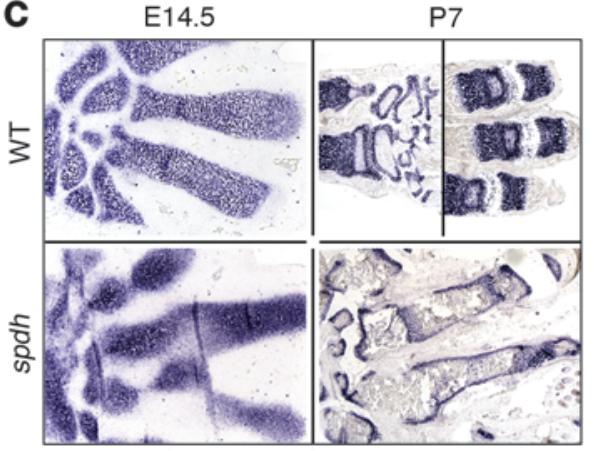

spdh
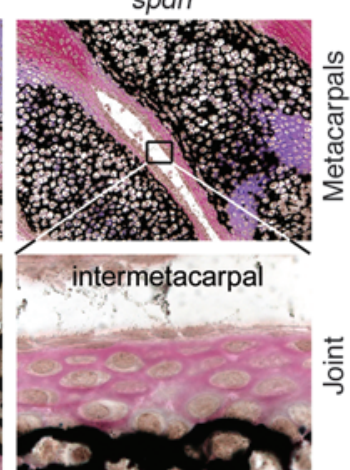

SPD patient

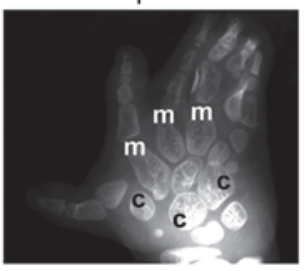

E

E12.5

E13.5

E14.5

E16.5
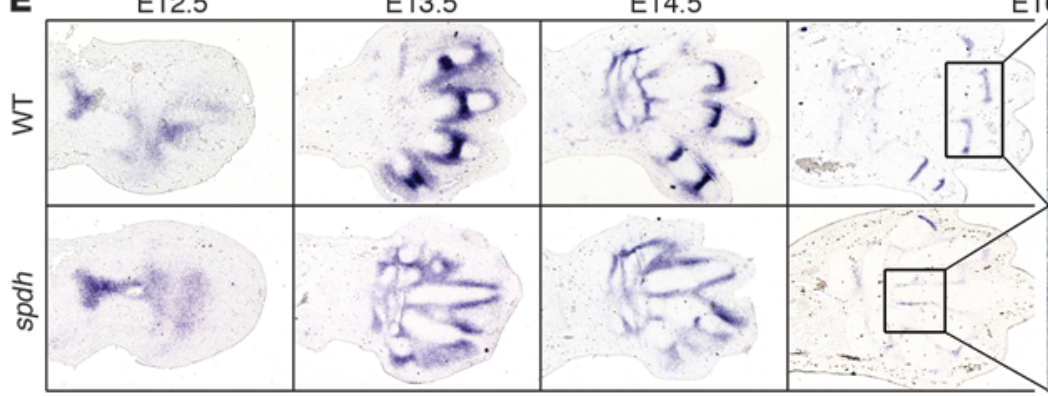

F
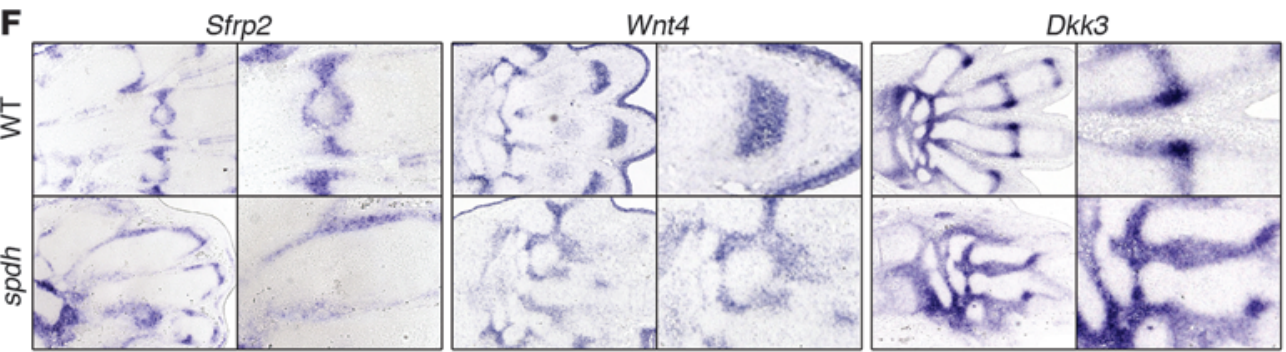

\section{Figure 4}

Homeotic transformation in Hox mutant mice. (A) Histology at P7 of WT and spdh/spdh forelimbs. Mutant metacarpal bones show mineralized cartilage (black) similar to WT carpal bones or secondary ossification centers. Mutant metacarpals have adopted a round carpal-like shape with joint-like gaps. c, carpal; cb, cortical bone; so, secondary ossification center. (B) Higher magnification $(\times 200)$ of metacarpal bones. WT cortical bone of metacarpals (black) is separated by connective tissue (pink). In mutant mice, carpal bones are surrounded by cartilage. This cartilage has a similar morphology to that in the joints of WT carpal bones. (C) In situ hybridization against Col2a1. At E14.5, Col2a1 is expressed throughout the cartilaginous anlage. At P7, WT (composited view) Col2a1 is expressed around the carpal bones and secondary ossification centers marking the joint cartilage. In spdh/spdh forelimbs, Col2a1 is expressed in a narrow band around the metacarpals similar to that seen in WT carpals. (D) $\mathrm{X}$-rays of a patient with synpolydactyly. Carpals (c) have a round shape; metacarpals $(\mathrm{m})$ are long bones, with cortical bones in the shaft region. In the patient, metacarpal bones have adopted a carpal-like shape. (E) In situ hybridization of Gdf5 at E12.5 to E16.5. In spdh/spdh limbs, Gdf5 is expressed around the cartilage anlagen. Boxes show magnifications (×250). (F) In situ hybridizations at E14.5 with joint markers Sfrp2, Wnt4, and Dkk3. In all cases, joint expression is lost in spdh/spdh animals; instead, there is an upregulation in perichondrial regions along the metacarpal structures.
Runx2-dependent bone formation requires bone morphogenetic protein (Bmp) signaling (21). As the Bmp4 promoter was shown to be activated by Hoxd13 and Hoxa13 (22), we investigated which of the 5 ' Hox genes are able to upregulate Bmp4 expression in chMM. Bmp4 was induced by Hoxd13 and Hoxa13 (Figure 3F), but not by Hoxd11 and Hoxd12. Interestingly, Runx2 also had an upregulating effect on Bmp4 expression, suggesting that Runx2 and Bmp4 are coupled in a regulatory circuit. In the WT E14.5 autopod, Bmp4 is expressed in the perichondrium. In $s p d h / s p d h$ mice, $B m p 4$ was strongly diminished and restricted to the distal parts of the digit anlagen (Figure 3G).
Homeotic transformations of metacarpal to carpal bones. Figure 4A shows the ossification process in WT carpals, the secondary ossification centers of WT metacarpals, and $s p d h / s p d h$ carpals and metacarpals. Cortical bone was present in the WT metacarpal bone shaft only. There was no difference between the ossification patterns of WT and $s p d h / s p d h$ carpals. In contrast, $s p d h / s p d h$ metacarpals showed a pattern similar to that of WT carpals and the secondary ossification center of the metacarpals. Furthermore, the overall shapes of $s p d h / s p d h$ metacarpals were more carpal-like. Thus, the ossification of $s p d h / s p d h$ metacarpals appeared to follow a secondary ossification 
scheme both in time and morphology (Supplemental Figure 3). In WT long bones, perichondrial cells are oriented in parallel layers around the cartilage anlage. The cells that surround carpal bones lack this polarity at early stages. This is similar to the spdh metacarpal region. Later, at E16.5, the WT carpal cells adjacent to the joint space became flattened, but they showed tangential instead of perpendicular orientation in comparison with the axis of the inner cells. This difference in lineage determination was accompanied by differences in the expression of Runx2, Bmp4, and osteopontin, which are highly expressed in the perichondrium of metacarpals and phalanges but not in the carpals (Supplemental Figure 4).

Histological examination showed minimal amounts of tissue between the $s p d h / s p d h$ metacarpals. In contrast, the area between 2 WT metacarpals was filled with periosteum and connective tissue. In $s p d h / s p d h$ mice, the mineralized hypertrophic cartilage was covered by cartilage that resembled the joint surface of carpals (Figure 4B). Expression analysis of Col2a1, the cartilage-restricted collagen, showed expression throughout the cartilaginous anlagen in WT and mutant digits at E14.5 (Figure 4C). At P7, Col2a1 expression was restricted in WT mice to the growth plates and the joints of the carpal and metacarpal bones. In $s p d h / s p d h$ mice, Col2a1 expression was present around the metacarpal bones, giving them a carpal-like appearance.

To determine whether early markers of joint development were expressed in these ectopic joints, we performed in situ hybridization with probes for growth and differentiation factor 5 (Gdf5), secreted frizzled-related protein 2 (Sfrp2), Wnt4, and dickkopf homolog 3 (Dkk3). In $s p d h / s p d h$ mice, the initial pattern of Gdf5 expression at E12.5 was similar to that of WT mice, but it did not change with time, i.e., it remained around the cartilage anlagen. Even at late stages (E16.5), we detected Gdf5 expression between the metacarpals (Figure 4E). Similar results were obtained for $S f r p 2$, Wnt4, and $D k k 3$, indicating that joint cartilage was specified at the wrong locations, i.e., not at the ends of the bones, but at their sides (Figure 4F). Besides being detected in the metacarpo-phalangeal joints, Dkk3 was found strongly expressed around the carpals and downregulated in the perichondrium. In $s p d h / s p d h$ mice, this pattern was lost and expression was observed not only around the carpals but also surrounding the metacarpals, indicating a lack of downregulation in the perichondrium. These results indicate that $s p d h / s p d h$ metacarpals had adopted a fate similar to that of carpal bones.

An x-ray of a normal human hand (Figure 4D) illustrates the characteristic ossification pattern of metacarpals, with thick cortical bone, a bone marrow cavity, and trabecular bone with adjacent joints at the ends. In contrast, the carpal bones, located between the metacarpals and radius/ulna, are round in shape and have a trabecular ossification pattern. The $x$-ray of an individual with a homozygous HOXD13 +9 Ala mutation (Figure 4D) shows metacarpal bones lacking their typical longitudinal pattern. Instead, they have a round shape and an ossification pattern that is similar to that of carpal bones.

Molecular pathology of the spdh mutation. Spdh/spdh mice have a different and more severe phenotype than Hoxd13-knockout mice, indicating that the $s p d h$ allele is not a loss of function but rather exerts a neomorphic and/or antimorphic effect. To test the possibility that mutant Hoxd13 might interact with and partially inactivate Hoxa13, we performed in vitro overexpression experiments with WT and mutant Hoxd13 together with Hoxa13. Mutant Hoxd13 forms intracellular aggregates that prevent the protein from entering the nucleus (11). Coexpression of mutant (+14 Ala) Hoxd13 with WT Hoxa13 resulted in the sequestering of Hoxa13 in the Hoxd13 aggregates (Figure 5A). The same effect was observed with other polyalanine-containing transcription factors such as Runx2 (Figure 5A). To investigate the specificity of the interaction of mutant Hoxd13 with WT Hoxa13, we performed a series of coexpression experiments. Coexpression of mutant Hoxd13 with WT Hoxd12 did not change its nuclear localization (Figure 5B). Coexpression of mutant Hoxd13 with WT Hoxd13, however, resulted in accumulation of WT protein in the aggregates. To determine whether the observed effect was due to the polyalanine tract, which is present in Hoxd13 and Hoxa13, but not in Hoxd12, we constructed a mutant version of Hoxd 13 with only 2 alanines ( 2 Ala), replacing the WT 15 alanine stretch. In contrast to the WT, this version of Hoxd13 (2 Ala) was found in the nuclei only, indicating that the polyalanine stretch was essential for the observed cytoplasmic sequestering effect.

We next tested to determine whether other transcription factors with polyalanine expansions as they occur in hand-foot-genital syndrome (HOXA13) (23) and cleidocranial dysplasia (RUNX2) (24) behave in a similar way (Figure 5C). Coexpression of mutant HOXA13 (+10 Ala) together with WT Hoxd13 resulted in the formation of aggregates that contained both proteins. Coexpression of mutant RUNX2 (+10 Ala) with WT Hoxd13 had the same effect, indicating that expanded alanine repeats have a general tendency to interact with other polyalanine-containing proteins, resulting in their sequestration in cytoplasmic aggregates.

To investigate the level of Hoxa13 in vivo, we performed immunohistochemistry with a Hoxa13-specific antibody (Figure 5D). We obtained nuclear staining in the autopod, but not in other regions of the limb. In WT mice, staining was found mainly in interdigital spaces and the perichondrium and localized to the nucleus. In the $s p d h / s p d h$ mutant, staining intensity was reduced and diffuse. Higher magnification showed that Hoxa13 protein was mainly present in the cytoplasm with less staining in the nuclei. These results indicate a reduced level of active Hoxa13 protein in $s p d h / s p d h$ limbs. Misfolding and aggregation of proteins is frequently associated with degradation and has also been observed for Hoxd13 in spdh/spdh limbs (11) We therefore tested the amount of Hoxa13 protein in $s p d h / s p d h$ limbs at E13.5 via Western blot. As illustrated in Figure 5E, $s p d h / s p d h$ limbs showed a reduction of Hoxa13 protein when compared with WT limbs.

\section{Discussion}

One important aspect of limb development is the establishment of a system of signaling molecules that determine the anteroposterior, proximodistal, and dorsoventral axes of the limb. This system ultimately controls the formation of skeletal precursor elements, which are laid down in a proximodistal fashion. A continuous challenge in the field of limb development research has been the question of where Hox genes fit into the molecular-genetic program of patterning and organogenesis of such elements. HoxA and HoxD genes belonging to the same paralogous group show similar expression patterns (5). During an early phase, Hox genes are involved in the regulation of anterior-posterior polarity through the control of Shb expression, the mediator of polarity in the limb (25). This regulation is likely controlled by the binding of Hox genes to a limb regulatory region upstream of the Shb gene (26). The function of Hox genes at a later phase of limb development is less well defined but is likely to involve the regulation of cell differentiation. Recently, we were able to show that Hox genes suppress chondrogenesis directly and indirectly by regulating retinoic acid concentration in the limb bud (27). These studies indicate that Hox genes not only have a role in early patterning processes but are 
A
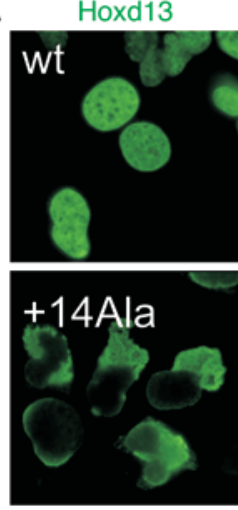

Hoxd13
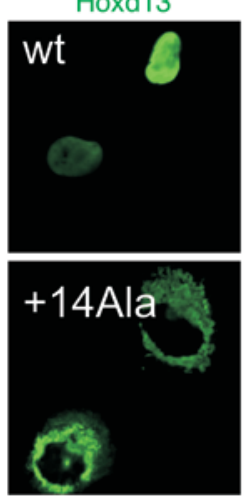

C

Hoxd13

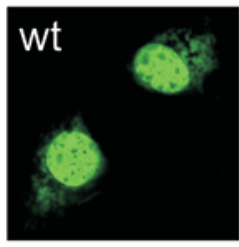

Hoxa13
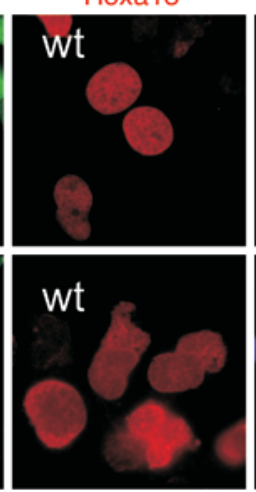

Runx2
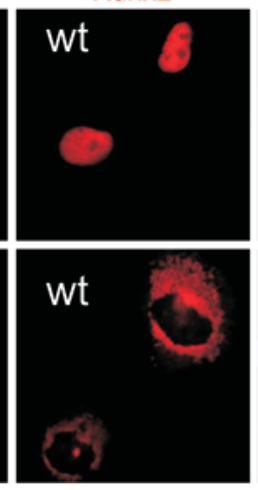

Merge
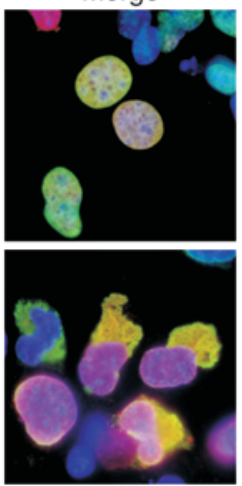

Merge

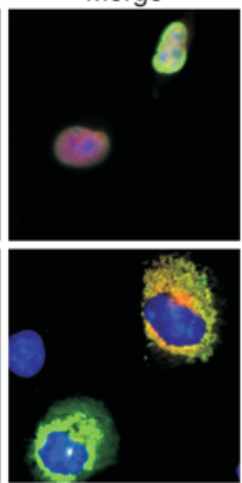

B

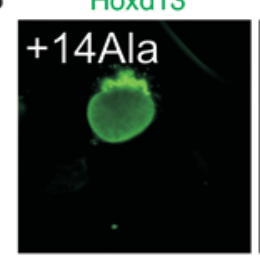

Hoxd13
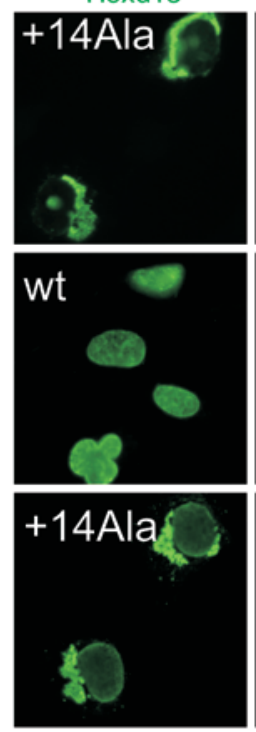

Hoxd12

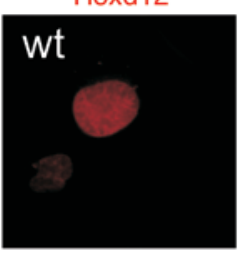

Hoxd13
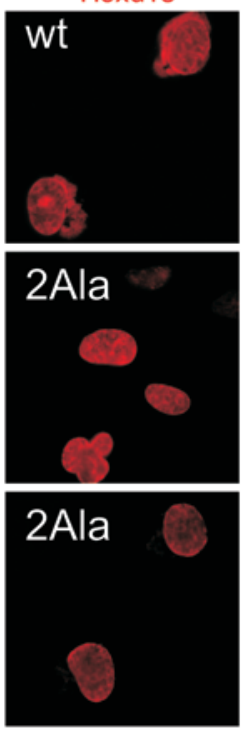

Merge

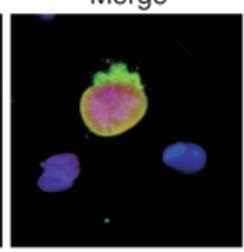

Merge
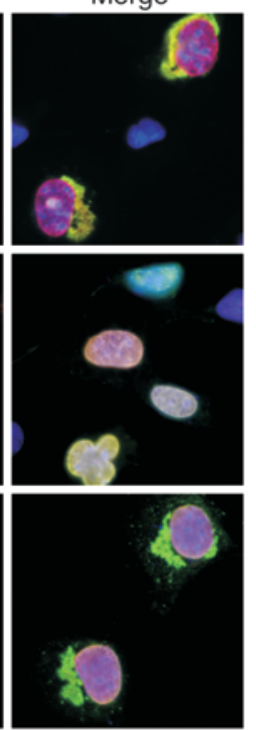

Hoxd13

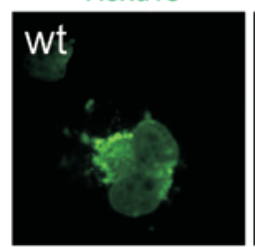

Runx2

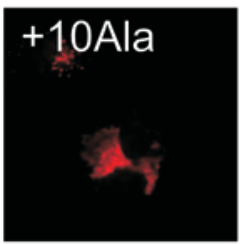

Merge

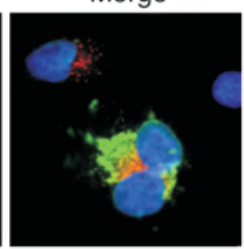

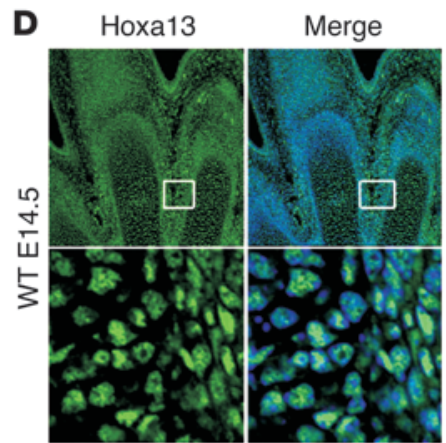
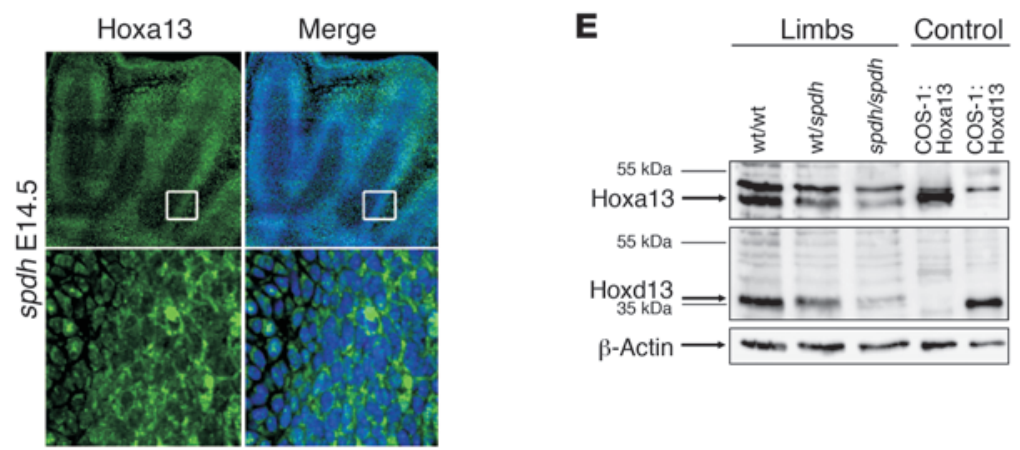

Figure 5

The dominant negative nature of polyalanine expansions. (A) WT Hoxd13 and WT Hoxa13 colocalize in the nucleus; expression of mutant Hoxd13 (+14 Ala) results in cytoplasmic aggregates. Coexpression of mutant Hoxd13 with WT Hoxa13 results in localization of Hoxa13 in aggregates. The same is true when coexpressing mutant Hoxd13 with Runx2 (lower panels). (B) Coexpression of Hoxd13 mutant with Hoxd13 WT, but not with Hoxd12 WT, results in sequestration of the WT protein in aggregates. Coexpression of mutant Hoxd13 with Hoxd13 with reduced alanine stretch (2 Ala) results in localization of the 2 Ala Hoxd13 in the nucleus. (C) Mutant Hoxa13 (+10 Ala) as well as mutant Runx2 (+10 Ala) form aggregates in cytoplasm. Coexpression with WT Hoxd13 results in localization of Hoxd13 in aggregates. (D) Immunohistochemistry of WT and spdh/spdh limbs at E14.5. Hoxa13 protein (green) is localized in nuclei of perichondrial cells. In spdh/spdh mice Hoxa13 protein shows an abnormal distribution with reduced intensity and cytoplasmic localization. Boxes mark magnifications ( $\times 630$ ) of the perichondrial regions of metacarpals. Original magnification, $\times 630$ (A-C); $\times 200$ (D). (E) Western blots on limb lysates of WT and spdh/spdh limbs at E13.5 showing a reduction of Hoxa13 as well as Hoxd13 protein. 
also important for cell differentiation. The data presented in this study indicate that Hox genes have an instructive role in determining the shape and ossification patterns of bones in the limbs.

The formation of all long bones starts with a cartilaginous model (anlage) of the future bone, which is subsequently replaced by bone. The formation of joints is an important step in this process, as it results in the persistence of cartilage in the articulating region in contrast to the rest of the bone. The signaling molecule Gdf5 has been linked to joint development, and lineage experiments have shown that joint cells originate from Gdf5-positive precursors (28). During early development (E12.0 in the mouse), Gdf5 expression is found in the interdigital mesenchyme surrounding the cartilaginous anlagen. Shortly thereafter, Gdf5 expression moves toward the future joints and is now found in a band of cells oriented perpendicular to the cartilage anlagen. In $s p d h / s p d h$ mice, this shift of Gdf5 expression does not take place. Instead, $G d f 5$ and other joint-expressed genes, such as Sfrp2, Wnt4, and Dkk3, continue to be expressed around the cartilage anlagen similar to the situation in the case of carpal bone anlagen.

After the initial process of mesenchymal condensation and chondrogenesis, which results in the formation of the cartilage anlagen, a specialized tissue is formed, the perichondrium. Whereas chondrocytes are oriented perpendicular to the long axis of the skeletal elements, perichondrial cells are parallel to this axis. Interdigital mesenchymal cells have, in contrast, no apparent orientation. We investigated $s p d h / s p d h$ mutants and show that neither the orientation of cells nor the expected change in gene expression takes place. Instead, $s p d h / s p d h$ cells adjacent to the cartilage anlage stay in a nonoriented undifferentiated stage. Matrix molecules such as collagen type 1, Crabp1, and Tsp2 as well as signaling molecules such as Wnt5a are expressed at very low levels, indicating that the cells fail to develop into perichondrial cells. On the other hand, genes such as Dkk3 that are expressed in the joints and that are consequently downregulated in the perichondrium are expressed at high levels around the metacarpal anlagen in $s p d h / s p d h$ mice. We hypothesize that this absence of a functioning perichondrium influences the chondrocytes in the cartilage anlage. Indeed, these cells have been shown to have low proliferation rates, do not hypertrophy, and do not become fully differentiated (9).

Cortical bone is generated by perichondrial precursor cells that differentiate into osteoblasts. Runx2 has been shown to be essential for this step, as Runx2-knockout mice do not form bone at all (3). We show here that Hoxd13 is a positive regulator of Runx2 expression. A regulation of Runx2 by homeobox genes has been postulated before. Muscle segment homeobox gene 2 (Msx2), distal-less homeobox gene $5(D l x 5), D l x 3$, and Hoxa10 were shown to regulate the activity of Runx2, indicating that a network of homeobox genes is involved in the control of Runx2 expression $(19,29,30)$. Our results support this hypothesis and show that Hox deficiency results in deficient bone formation not only in vitro but also in vivo. The effect of Hox on Runx2 is likely to be enhanced by the regulation of Bmps, in particular Bmp4, by Hox genes. Hoxd13 mutants with elongated polyalanine repeats lose their ability to activate the $B m p 4$ promoter (data not shown), and $s p d h / s p d h$ mutant mice show a diminished expression of Bmp4. Reduced levels of Bmps will, in turn, result in a further reduction of Runx2 and also Osterix expression, as both transcription factors are downstream targets of the Bmp pathway (29).

Homeosis, the transformation of one body part into another, is associated with mutations in or misexpression of Hox genes. In Drosophila, examples of such mutations are antennapedia and the bithorax series of mutations. Homeotic transformations have also been observed in mice. Here, inactivation of certain Hox genes or all paralogous groups results in a change in the identity of one or many vertebrae into the identity of others $(6,15,31)$. Unlike the situation in the trunk, loss-of-function phenotypes in the limbs have not been interpreted as classical homeotic transformations (5). We propose that the inactivation of Hox genes in the autopod affects growth and differentiation of cartilaginous elements in such a way that the result is homeotic transformation of metacarpal bones.

The results of our study show that metacarpals lose their identity as long bones and are transformed into carpal-like bones. This transformation is accompanied by a change in the 3 major attributes of long bones, i.e., the presence of cortical bone, the position of the joints, and the shape of the bone. In long bones, the joints are positioned at the ends, the cortical bone is at the midshaft region, and the shape is that of a cylinder with cartilaginous ends. In round bones, such as the carpals, there is no cortical bone, joints surround the bone, and the shape is that of an ovoid disc. The absence of cortical bone in carpals also determines their pattern of ossification. In contrast to long bones that form primary ossification centers, carpal bones ossify through a process similar to secondary ossification. In addition, primary ossification of long bone cartilage models takes place during development (in the mouse E13.5), whereas secondary ossification happens at defined time points after birth. Here we show that the pattern of ossification and the time of ossification of mutant metacarpals are similar if not identical to those of the secondary ossification centers of WT carpals and long bones. Thus, the spdh metacarpals fulfill all criteria of ovoid, carpal bones, arguing that a transformation of one body part into another and thus a homeotic transformation has taken place. The observed change in identity cannot be explained by the lack of bone formation or the defect in chondrocyte differentiation. Inactivation of $I h h$, for example, results in a complete absence of bone and severely disturbed chondrocyte differentiation. In spite of this, the long bones of $\mathrm{Ihb}^{-/-}$mice do not become round; neither are they surrounded by joints. They retain their original shape, while being shorter (32). The same holds true for Runx2-/ mice, in which, despite a complete absence of bone, the cartilage anlagen do not change their overall shapes, each element being easily recognizable (3). This is most likely due to the relatively late effect of Runx2, which exerts its major role in the specification of osteoblasts and the regulation of differentiation of precursor cells into this lineage. Hox genes, in contrast, act much earlier and are able to regulate other genes in this pathway such as the BMPs. As we had previously reported (9), BMPs are regulated by Hoxd13 before Runx2 starts to be expressed. This indicates that Hox genes organize and dictate the emergence of the perichondrium and that Runx2 is a downstream player.

We observed a similar effect in Hoxd11,12,13 del/del, as well as in Hoxd13 $3^{-1-}$; Hoxa $13^{+/-}$mice, as the metacarpals of these mutants also do not have cortical bone and ossify according to the secondary ossification scheme. Thus, Hox genes of the $5^{\prime} \mathrm{D}$ and A cluster determine the shape and identity of autopod metacarpal skeletal elements. Patients that are homozygous for polyalanine expansion mutations have a severe limb phenotype consisting of polydactyly and severe brachydactyly $(8,33)$. In these individuals, the metacarpals also lose their identity of long bones and become round, carpal-like bones. On $\mathrm{x}$-ray this transformation is evident, as these bones do not show the clear distinction between cortical and trabecular bone as observed in WT metacarpals. Furthermore, surgical reports indicate that these bones are covered with cartilage, thus giving them a joint-like appearance (34).

Polyalanine repeats are relatively common in transcription factors, but their functional role remains unclear. The length of the 
glutamine/alanine repeat in Runx2 was shown to affect transcriptional activity and was proposed to be involved in the evolution of facial length in carnivores $(35,36)$. In Hoxd13, a novel expansion of the polyalanine tract was identified in cetaceans (whales, dolphins) compared with other mammals, indicating a role in the morphological diversity of the cetacean autopod (37). Polyalanine mutations can be classified as a separate class of repeat expansions associated with developmental malformations. With one exception, all polyalanine repeat mutations have been reported in transcription factors (38). For most polyalanine mutations, loss of function due to protein degradation seems to be the major pathogenic mechanism (11). In Hoxd13, however, the size of the polyalanine expansion correlates with the severity of the disease (39), and recent studies have shown that expansion of polyalanine repeats in Hoxd13 results in a combination of loss of function and gain of function (27). The latter involves a negative effect on other Hox genes (10).

In this study, we have addressed the molecular basis of this obser-

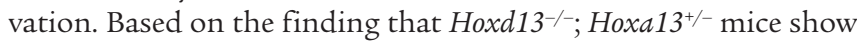
changes similar to those of the $s p d h / s p d h$ mutant mice, we tested the hypothesis that mutant Hoxd13 might interact with WT Hoxa13. Based on colocalization of Hoxa 13 with Hoxd $13^{+14 a l a}$ in cytoplasmic aggregates, abnormal distribution of Hoxa13 protein in $s p d h / s p d h$ limbs, and a downregulation of Hoxa13 protein in $s p d h / s p d b$ limbs, we propose that mutant Hoxd13 associates with WT Hoxa13, which results in a degradation not only of the mutant Hoxd13 but also of WT Hoxa13. Furthermore, we show that this interaction is likely to be mediated via the polyalanine stretch. This explains why other Hoxd genes, such as Hoxd12, which lack a polyalanine stretch do not colocalize with the mutant Hoxd13 protein (11).

In addition, our findings suggest that mutant Hoxd 13 is able to interact with other proteins that carry polyalanine repeats, such as Runx2. This is likely to increase the negative effect of mutant Hoxd13 on Runx2. Furthermore, the observation that transcription factors with expanded alanine repeats bind to other polyalanine-containing proteins offers a new understanding of the pathogenesis of polyalanine repeat-related diseases. It may also explain clinical features that are not considered part of the classical phenotype. For example, in a family with a polyalanine expansion in RUNX2, brachydactyly was observed in addition to cleidocranial dysplasia, the known phenotype associated with RUNX2 haploinsufficiency (24). Brachydactyly has also been observed as a consequence of mutations in HOXD13, indicating a double effect (loss of HOXD13 and RUNX2 function) in affected individuals.

\section{Methods}

Patients. The patient with synpolydactyly is from Pakistan. X-ray examination was performed for diagnostic reasons. DNA was extracted with standard methods from peripheral blood. Sequencing of HOXD13 was performed as described previously (8). The patient gave written informed consent.

Mice. Spdh/+ mice were obtained from Jackson Laboratory (40). Mutant offspring were generated by breeding $s p d h /+$ females to $s p d h /+$ males. Mutant mice with conditional knockout of Hoxd11,12,13 del/del in the limbs were generated by crossing mice carrying flox (flanked by loxP sites) alleles of Hoxd11,12,13 with transgenic mice expressing cyclization recombinase under the control of periaxin 1 promoter (Prx1-Cre). On the day of vaginal plug, embryonic stage at $12 \mathrm{pm}$ was considered to be E0.5. Genotyping was performed as described previously (27), using DNA from tail tip, amnion, or liver. Animals were analyzed by skeletal preparations as described previously (41). WT littermates served as controls. All animal experiments were approved by the Landesamt für Gesundheit und Soziales.
Immunohistochemistry, histology, and in situ bybridization. Limbs were embedded in paraffin and plastic according to standard procedures. Immunohistochemistry was performed on $7-\mu \mathrm{m}$ paraffin limb sections. Further procedures were performed as previously described (27). Rabbit anti-Hoxa13 (\#ARP31955_P050; Aviva Systems Biology), rabbit antiHoxd13 (gift from Atsushi Kuroiwa, Division of Biological Sciences, Nagoya University, Nagoya, Japan), and rabbit anti-collagen I (ab21286; Biozol) were used as primary antibodies. Alexa Fluor 488-conjugated goat anti-rabbit (Invitrogen) was used as secondary antibody. Micrographs were taken with an AxioVert 200 fluorescence microscope (Zeiss). In situ hybridization was performed as described previously (42).

chMM culture. chMM cultures were prepared as described previously (43). Cells were infected with concentrated viral supernatants containing the cDNA encoding WT Hoxd13, Hoxd13 $3^{+7 \text { Ala }}$, Hoxd13 ${ }^{+14 A l a}$, Hoxd11, Hoxd12, and Hoxa13. Infection with empty RCASBP virus (44) was used as a control. For each condition, quadruplicate micromasses were cultivated in parallel. qPCR was performed as previously described (45) with gene-specific primers.

Immunofluorescence. Cos-1 (CV-1 origin, SV40) cells were cultured, transfected, and incubated with antibodies as previously described (11).

Western blot. For Western blot, E13.5 limbs of WT and $s p d h$ were lysed by $50 \mathrm{mM}$ HEPES buffer supplemented with protease inhibitors and homogenized by shearing with a $1-\mathrm{ml}$ syringe. Membranes were probed with the following antibodies: 1:1000 rabbit anti-Hoxa13 (\#ARP31955_P050; Aviva Systems Biology) and 1:8000 rabbit anti-Hoxd13 (gift from Atsushi Kuroiwa). Protein visualization on Western blot membrane was performed by chemiluminescence (Roth) after incubation with 1:1000 peroxidase-conjugated goat-anti-rabbit (Oncogene) antibody. Specific signals were quantified using a Western blot imager.

ChIP and luciferase reporter assay. RCASBP virus-infected chMM cultures expressing an $\mathrm{N}$-terminal FLAG-tagged Hoxd 13 were cultured for 9 and 12 days. ChIP procedures were performed as previously described (27). Primers were as follows: P1 forward, TATATCGCTGTGGATGCCAGG; P1 reverse, TTTTGACAAAAGGCCCGTG; P2 forward, GTGTGAATGCTTCATTCGCCT; P2 reverse, ACCGCACTTGTGGTTCTGTG; P3 forward, GGTTAAGCTCCGCAGGTCAC; P3 reverse, TTACTGACTCTGTTGGTCTCAGTGG; P4 forward, CAGCCACTGAGACCAACAGA; P4 reverse, TGTGAAAAGCGAGGAAACAA. For luciferase reporter assay, a murine Runx 2 promoter region from -421 bp to +122 bp (based on GenBank AF005936) was amplified from genomic mouse DNA with specific primers GATCGGTACCGCTATAACCTTCTGAATGCCAG (forward) and GATCGGATCCTGCATGGACTGTGGTTA (reverse) and cloned into pGL3-basic luciferase reporter vector. Mutation of putative Hox-binding sites was performed using the following primers: for mutation of binding site 1 at position -380, CTTACCACAAGCCTGTGGTCAGAGGGAGAAAGG (forward) and CСТTTCTCCСТCTGACCACAGGCTTGTGGTAAG (reverse); for mutation of binding site 2 at position -90, CAGTAATAGTGCTTGCACACACTAGGAGTTTTAAAGC (forward) and GCTTTAAAACTCCTAGTGTGTGCAAGCACTATTACTG (reverse); for mutation of binding site 3 at position -78, CTTGCAAAAAATAGGAGTGTGACAGCTTTTGCTTTTTTGG (forward) and CCAAAAAAGCAAAAGCTGTCACACTCCTATTTTTTGCAAG (reverse); for mutation of binding site 4 at position -63, GAGTTTTAAAGCTTTTGCTGTGTGGGATTGTGTGAATG (forward) and CATTCACACAATCCCACACAGCAAAAGCTTTAAAACTC (reverse); for mutation of binding site 5 at position +47, TCTGGCGTTTAAATGGTGACTCTCTGCAGGTCACTA (forward) and TAGTGACCTGCAGAGAGTCACCATTTAAACGCCAGA (reverse). Deletion of binding sites 2-4 was performed by PCR using primers flanking the putative binding sites and religation of the PCR product: GGATTGTGTGAATGCTTCATTCG (forward) and CAAGCACTATTACTGGAGAGACAG 
(reverse). The Runx2 promoter fragment in pGL3-basic was used as a template for mutagenesis PCRs. After mutagenesis, promotor fragments were excised by KpnI/NcoI and cloned into pGL3 basic. NIH/3T3 cells were transfected using PolyFect (QIAGEN) in 24-well plates with the reporter constructs $(250 \mathrm{ng} /$ well) together with an expression vector containing either the different Hox constructs or an empty vector as control (100 ng/well). pRL-CMV (5 ng/well) were cotransfected for normalization. Cells were lysed with $100 \mu$ l passive lysis buffer (Dual Luciferase Assay Kit; Promega). $5 \mu$ l of the lysate was measured using the Dual-Glo Luciferase Assay Kit (Promega) with $25 \mu \mathrm{l}$ of the assay reagents each. Measurements were performed on a 1450 MicroBeta Scintillation and Luminescence Counter (PerkinElmer).

Statistics. Graphs show mean \pm SD of triplicate samples.

\section{Acknowledgments}

We thank P. Chambon for kindly providing us with $\mathrm{Hoxa} 13^{+/-}$ mice. This work was supported by a grant from the Deutsche Forschungsgemeinschaft (DFG) to Stefan Mundlos.

Received for publication October 23, 2009, and accepted in revised form March 17, 2010.

Address correspondence to: Stefan Mundlos, Institute for Medical Genetics, Charité, Universitätsmedizin Berlin, Campus Virchow, Augustenburger Platz 1, 13353 Berlin, Germany. Phone: 49.30.450.569.121; Fax: 49.30.450.569.915; E-mail: stefan. mundlos@charite.de.
1. Karsenty G. The complexities of skeletal biology. Nature. 2003;423(6937):316-318.

2. Kornak U, Mundlos S. Genetic disorders of the skeleton: a developmental approach. Am J Hum Genet. 2003;73(3):447-474.

3. Otto F, et al. Cbfa1, a candidate gene for cleidocranial dysplasia syndrome, is essential for osteoblast differentiation and bone development. Cell. 1997;89(5):765-771.

4. Lewis EB. The 1991 Albert Lasker Medical Awards. Clusters of master control genes regulate the development of higher organisms. JAMA. 1992; 267(11):1524-1531.

5. Zakany J, Duboule D. The role of Hox genes during vertebrate limb development. Curr Opin Genet Dev. 2007;17(4):359-366.

6. Wellik DM, Capecchi MR. Hox10 and Hox11 genes are required to globally pattern the mammalian skeleton. Science. 2003;301(5631):363-367.

7. Zakany J, Fromental-Ramain C, Warot X, Duboule $D$. Regulation of number and size of digits by posterior Hox genes: a dose-dependent mechanism with potential evolutionary implications. Proc Natl Acad Sci U S A. 1997;94(25):13695-13700.

8. Muragaki Y, Mundlos S, Upton J, Olsen BR. Altered growth and branching patterns in synpolydactyly caused by mutations in HOXD13. Science. 1996;272(5261):548-551.

9. Albrecht AN, Schwabe GC, Stricker S, Boddrich A, Wanker EE, Mundlos S. The synpolydactyly homo$\log$ (spdh) mutation in the mouse - a defect in patterning and growth of limb cartilage elements. Mech Dev. 2002;112(1-2):53-67.

10. Bruneau S, Johnson KR, Yamamoto M, Kuroiwa A, Duboule D. The mouse Hoxd13 (spdh) mutation, a poly-alanine expansion similar to human type II synpolydactyly (SPD), disrupts the function but not the expression of other Hoxd genes. Dev Biol. 2001;237(2):345-353.

11. Albrecht AN, et al. A molecular pathogenesis for transcription factor associated poly-alanine tract expansions. Hum Mol Genet. 2004;13(20):2351-2359.

12. Davis AP, Capecchi MR. A mutational analysis of the 5' HoxD genes: dissection of genetic interactions during limb development in the mouse. Development. 1996;122(4):1175-1185.

13. Dolle P, et al. Disruption of the Hoxd-13 gene induces localized heterochrony leading to mice with neotenic limbs. Cell. 1993;75(3):431-441.

14. Zakany J, Duboule D. Synpolydactyly in mice with a targeted deficiency in the HoxD complex. Nature. 1996;384(6604):69-71.

15. Fromental-Ramain C, Warot X, Messadecq N, LeMeur M, Dolle P, Chambon P. Hoxa-13 and Hoxd13 play a crucial role in the patterning of the limb autopod. Development. 1996;122(10):2997-3011.
16. Rios H, et al. Periostin null mice exhibit dwarfism, incisor enamel defects, and an early-onset periodontal disease-like phenotype. Mol Cell Biol. 2005;25(24):11131-11144.

17. Witte F, Dokas J, Neuendorf F, Mundlos S, Stricker S. Comprehensive expression analysis of all Wnt genes and their major secreted antagonists during mouse limb development and cartilage differentiation. Gene Expr Patterns. 2009;9(4):215-223.

18. Bandyopadhyay A, Kubilus JK, Crochiere ML, Linsenmayer TF, Tabin CJ. Identification of unique molecular subdomains in the perichondrium and periosteum and their role in regulating gene expression in the underlying chondrocytes. Dev Biol. 2008;321(1):162-174.

19. Hassan MQ, et al. HOXA10 controls osteoblastogenesis by directly activating bone regulatory and phenotypic genes. Mol Cell Biol. 2007;27(9):3337-3352.

20. Berger MF, et al. Variation in homeodomain DNA binding revealed by high-resolution analysis of sequence preferences. Cell. 2008;133(7):1266-1276.

21. Phimphilai M, Zhao Z, Boules H, Roca H, Franceschi RT. BMP signaling is required for RUNX2dependent induction of the osteoblast phenotype. J Bone Miner Res. 2006;21(4):637-646.

22. Suzuki M, Ueno N, Kuroiwa A. Hox proteins functionally cooperate with the GC box-binding protein system through distinct domains. J Biol Chem. 2003;278(32):30148-30156.

23. Mortlock DP, Innis JW. Mutation of HOXA13 in hand-foot-genital syndrome. Nat Genet. 1997; 15(2):179-180.

24. Mundlos S, Olsen BR. Heritable diseases of the skeleton. Part I: Molecular insights into skeletal development-transcription factors and signaling pathways. FASEB J. 1997;11(2):125-132.

25. Tarchini B, Duboule D, Kmita M. Regulatory constraints in the evolution of the tetrapod limb anteriorposterior polarity. Nature. 2006;443(7114):985-988.

26. Capellini TD, et al. $\mathrm{Pbx} 1 / \mathrm{Pbx} 2$ requirement for distal limb patterning is mediated by the hierarchical control of Hox gene spatial distribution and Shh expression. Development. 2006;133(11):2263-2273.

27. Kuss $P$, et al. Mutant Hoxd13 induces extra digits in a mouse model of synpolydactyly directly and by decreasing retinoic acid synthesis. J Clin Invest. 2009;119(1):146-156.

28. Koyama E, et al. A distinct cohort of progenitor cells participates in synovial joint and articular cartilage formation during mouse limb skeletogenesis Dev Biol. 2008;316(1):62-73.

29. Hassan MQ, et al. BMP2 commitment to the osteogenic lineage involves activation of Runx 2 by DLX3 and a homeodomain transcriptional network. J Biol Chem. 2006;281(52):40515-40526.

30. Shirakabe K, Terasawa K, Miyama K, Shibuya H,
Nishida E. Regulation of the activity of the transcription factor Runx2 by two homeobox proteins, Msx2 and Dlx5. Genes Cells. 2001;6(10):851-856.

31. Davis AP, Capecchi MR. Axial homeosis and appendicular skeleton defects in mice with a targeted disruption of hoxd-11. Development. 1994; 120(8):2187-2198.

32. St-Jacques B, Hammerschmidt M, McMahon AP. Indian hedgehog signaling regulates proliferation and differentiation of chondrocytes and is essential for bone formation. Genes Dev. 1999;13(16):2072-2086.

33. Akarsu AN, Akhan O, Sayli BS, Sayli U, Baskaya G, Sarfarazi M. A large Turkish kindred with syndactyly type II (synpolydactyly). 2. Homozygous phenotype? J Med Genet. 1995;32(6):435-441.

34. Kuru I, Samli H, Yucel A, Bozan ME, Turkmen S, Solak M. Hypoplastic synpolydactyly as a new clinical subgroup of synpolydactyly. J Hand Surg Br. 2004; 29(6):614-620

35. Fondon JW 3rd, Garner HR. Molecular origins of rapid and continuous morphological evolution. Proc Natl Acad Sci U S A. 2004;101(52):18058-18063.

36. Sears KE, Goswami A, Flynn JJ, Niswander LA. The correlated evolution of Runx2 tandem repeats, transcriptional activity, and facial length in carnivora. Evol Dev. 2007;9(6):555-565.

37. Wang Z, et al. Adaptive evolution of 5'HoxD genes in the origin and diversification of the cetacean flipper. Mol Biol Evol. 2009;26(3):613-622.

38. Albrecht A, Mundlos S. The other trinucleotide repeat: poly-alanine expansion disorders. Curr Opin Genet Dev. 2005;15(3):285-293.

39. Goodman FR, et al. Synpolydactyly phenotypes correlate with size of expansions in HOXD13 poly-alanine tract. Proc Natl Acad Sci U S A. 1997; 94(14):7458-7463.

40. Johnson KR, Sweet HO, Donahue LR, Ward-Bailey $\mathrm{P}$, Bronson RT, Davisson MT. A new spontaneous mouse mutation of Hoxd13 with a poly-alanine expansion and phenotype similar to human synpolydactyly. Hum Mol Genet. 1998;7(6):1033-1038.

41. Mundlos S. Skeletal morphogenesis. Methods Mol Biol. 2000;136:61-70.

42. Stricker S, Fundele R, Vortkamp A, Mundlos S. Role of Runx genes in chondrocyte differentiation. Dev Biol. 2002;245(1):95-108.

43. Seemann P, et al. Activating and deactivating mutations in the receptor interaction site of GDF5 cause symphalangism or brachydactyly type A2. J Clin Invest. 2005;115(9):2373-2381.

44. Federspiel MJ, Hughes SH. Retroviral gene delivery. Methods Cell Biol. 1997;52:179-214.

45 . Hecht J, et al. Detection of novel skeletogenesis target genes by comprehensive analysis of a Runx2(-/-) mouse model. Gene Expr Patterns. 2007; $7(1-2): 102-112$. 